\title{
Extracting Control Pulses of Handwriting Movement
}

\section{— Toward an Active Recognition of Cursive Handwritten Characters -}

\author{
Youssef IGUIDER* and Makoto YASUHARA*
}

\begin{abstract}
We propose an analysis method of human handwriting movements aiming at an active recognition of cursive handwritten characters. The analysis is performed by synthesizing signals according to a model and comparing the signals synthesized with the signals to be analyzed. To begin with, we will introduce the formal concept of the active recognition. And a model of human handwriting will be presented based on an Auto-Regressive Moving Average (ARMA) process by limiting discussions exclusively to the case of cursive handwriting. The model was first proposed by one of the authors in $1983^{7}$, and the extensive experimental studies had been made to reveal its utilities. Based on the ARMA model, the mathematical techniques to realize the active recognition will be given by showing that the model reference adaptive control system (MRACS) is able to be applied successfully to extract control signals of the cursive handwriting movements. The control signals are considered to play the most important role in the active recognition of the cursive scripts.
\end{abstract}

Key Words : cursive handwritten character recognition, inverse problem, active analysis/recognition, modeling, MRAC system

\section{Introduction}

Our studies are mainly stimulated by the need for on-line recognition of handwritten characters. The idea of using handwriting to interface with computers has been attracting attentions for many years. With the recent advances in devices and packaging technologies, a low-priced and unified tablet-display system having the size and weight of a note is becoming of reality. Electronic tablet accurately captures ( $x$, $y$ )-coordinate data of pencil-point movements, and electronic ink displays traces of the pencil-point movements on the screen. Flexibility is an important property of handwriting. Writing on the tablet with no constraints is just as writing with a pen on the paper.

On the other hand, the recognition algorithm that converts coordinate data into symbol codes has been remained for further studies. For handprinted character recognition, great many research works have been reported so far and already at the level of practical applications. However, it is generally agreed that the recognition problem of cursive handwritten characters is so difficult that it has been remained unsolved even for the on-line case, and that it is much more difficult for the off-line case and therefore has been almost untouched.

$\dagger$ Presented 24th Stocastic System Symposium $(1992 \cdot 11)$

* The University of Electro-Communications, 1-5-1, Chofugaoka, Chofu

(Received March 11, 1994)

(Revised October 19, 1994)
Basically, this paper stands at the first stage oriented to the recognition of cursive handwritten characters. To begin with, we introduce an active way of processing, the concept of an active recognition underlying our current studies, and propose its realization technique, more specifically, the method to extract signals that control the cursive handwriting movements, and give its mathematical basis.

\section{Active Recognition-fundamental Concept}

In 1969, K. N. Stevens ${ }^{1)}$ proposed an Analysis-bySynthesis $(\mathrm{A}-\mathrm{b}-\mathrm{S})$ technique in speech research and in 1980, A. M. Liberman ${ }^{2}$ presented a motor theory of speech perception. These two research works could be understood together to have argued that it is important for the speech recognition to extract information at the higher level of speech processing in the brain and thus to develop techniques to realize it. If a complete set of those informations for each pattern class can be extracted from the given data, the recognition task of the patterns will present little difficulty, that is, the task may be reduced to a simple matching process or a table look-up scheme. Although we have no space enough to give further discussions about these or related topics in depth, the thing we would like to point out here concerning our present studies is the following.

Writing and speech play the most important role in human communications. These two forms of verbal behavior have appreciable similarities as well as differences. Although their physical appearances are different and different sets of muscles are used in the 
execution of the appropriate gestures, the problems they present at the higher levels of language processing seem to be the same, that is, at some level of the language processing in the brain both must relate to the same grammatical structures of the language in question. Thus the processes corresponding to the generation or recognition of handwriting and to those of speech may be in a one-to-one relation at those levels. So, many of the problems concerning speech processing would probably be related closely to the corresponding problems concerning handwriting processing. Thus the Stevens's A-b-S scheme must play an essential part also in the handwriting processing.

One of the traditional and common cases for understanding handwritten characters is gathering and analyzing statistics related to their variations in shapes, slants, stroke numbers, orders, directions and so forth, which we have called as passive analysis. Another insight for understanding them may come from modeling human handwriting process and studying how to generate handwriting samples, which stem from the idea of the Steven's A-b-S. We call this as active analysis in contrast to the previous one.

Figure 1 shows the structure of human handwriting process assumed here, which we call handwriting hievarchy. We consider 4 basic levels of signal representations: (1) symbol, (2) motor command, (3) pencil-point movement and (4) handwritten character image. For generating handwriting characters, we make a forward trace from the top to the bottom level through the neurological and dynamical transformations to have the character image in the final form (the time-eliminated locus of pencil-point movements). Here we introduce the following fundamental assumption:

The active recognition of cursive handwritten char-
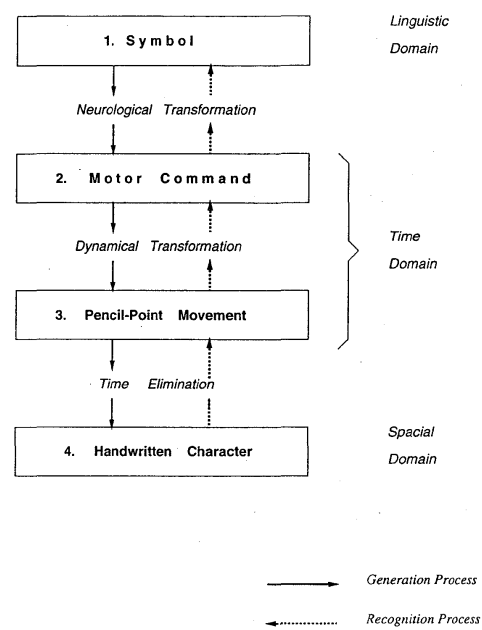

Fig. 1 Handwriting hierarchy acters can be seen as an inverted process of generation, that is, the process that makes an inverse trace of the handwriting hierarchy shown in Fig. 1 from the bottom to the top level. We say that the recognition system is active if and only if it does involve in itself some generation process of the patterns to be recog. nized, otherwise passive.

Note of course that for the recognition of handprinted characters a simple and straightforward method based on the passive analysis could successfully be applied, which converts the handwritten character directly to the corresponding symbol. In other words, it might not be necessary for the handprinted character recognition to apply such complicated method as tracing each of the whole levels of the handwriting hierarchy back from the bottom to the top level.

For the cursive handwritten character recognition, it follows from the above assumption that to solve three inverse problems in the sequence from the bottom to the top level results in to recognize the characters. By the inverse problem, we mean here the problem of extracting relevant information at one level of signal representations shown in Fig. 1 from that at the lower level. Generally, the inverse problem is difficult to solve because of its ill-posedness and therefore information deficiencies. One of the common and possible cases is to replace the inverse problem with its own forward one by introducing a generation model, and to apply some iterative approximation technique to have a solution in such a way that it minimizes the given error criterion. Above is the basic and key concept underlying our studies. For the purpose of references, in appendix A, there are comments on the studies concerning the generation of human handwriting movements.

In this paper, we propose a method to extract signals at the motor command level from those at the lower level, namely, at the level of pencil-point movements and give its mathematical basis. The model reference adaptive control system (MRACS) makes it possible for us to execute this extraction process. More specifically, the process is performed by generating signals according to the model and by comparing the generated signals with the signals to be analyzed. To begin with, we discuss about how an auto-regressive moving average (ARMA) process can be applied to model the human handwriting movements, and show that the MRACS can successfully be used for extracting control signals of the handwriting movements as well as to estimate the model parameters. The idea has stemmed basically from the recognition that the MRACS itself is equivalent in principle to the system of the active analysis in our sense, which will be discussed in the chapter 4 .

It has been reported recently by one of the authors 
et al. $^{3)}$ that it is possible to recover the pencil-point movement from the static handwritten character images. This means totally from the above discussions that we can trace the handwriting hierarchy back from the bottom to the motor command level shown in Fig. 1, that is, the cursive handwritten character recognition of the off-line type would be of reality if it becomes possible to trace from the motor command level back to the symbol level. Investigations are now under way in this direction.

\section{Modeling of Handwriting Process}

\subsection{Control Principle of Fast Cursive Hand- writing}

Though an important principle involved in controlling human handwriting movements is position feedback, it is well-known among physiologists that the principle does not always hold for fast or wellpracticed movements. Lashley ${ }^{4)}$ studied such processes and concluded that an effector mechanism could be pre-set to discharge at a given intensity or for a given duration, independent of any sensory control. According to Lashley, this holds also for the contraction of muscles in a complex movement such as fast cursive handwriting. His conclusions were based on the fact that the accuracy of a motion in terms of position was increased with velocity, and that the nerve delay was too long to correct the motion. For example, in the cursive handwriting movements, the speed of $50 \mathrm{~mm} / \mathrm{sec}$ of the pencil-point along the line of travel is common, thus the accuracy of $0.5 \mathrm{~mm}$, for instance, corresponds to $10 \mathrm{msec}$. Even spinal reflex time is not so short and nerve transmission time hand-head-hand is much longer. Dawson ${ }^{5)}$ gave an estimation of about $30 \mathrm{msec}$ for this nerve transmission time.

\section{2 Handwriting Model}

In this paper, by limiting our discussions to the case of the fast cursive handwriting, we consider a modeling of human handwriting process. By the fast cursive handwriting we mean highly practiced and skilled movements of handwriting which are executed by means of a rapid sequence of motions without instantaneous position feedback. To limit the discussions exclusively to the fast cursive handwriting enable us to introduce a simplified but satisfactory model of handwriting movement because, as discussed in the previous section, we need not consider any feedback process involved.

Figure 2 shows a pulse-controlled model of human handwriting process- the system theoretic representation. The system has no feedback involved at all and is supposed to be driven by the hypothetical input signal, control pulse, to give the handwriting signal at its output. Further, the system is considered to be divided into two subsystems, the muscular system $M$ and the hand system $H$.

\section{3 Control Pulse}

\subsection{Definition}

The movements involved in the actual handwriting are so much complicated that an attempt to interpret them in terms of a simplified model might be pointless, if we are to make precise physiological investigations about the actual human handwriting process. However, it must be rather contributory to our present studies to take the simplified model into account according to the discussions of the previous sections.

To begin with, we consider the following two assumptions $^{6)}$ : (1) the handwriting movements are resolved into three principal components, namely, $X$, $Y$ and $Z$ directions (the $Z$-component, writing pressure, is neglected just for simplification), (2) the muscles involved in each component of the movements are grouped together into a pair to cause them to generate the forces to antagonistic directions having the prescribed magnitude and timing's. Under these basic assumptions, we introduce the definition of control pulse, which plays an essential role in this study.

The cursive characters are written by a rapid sequence of motions caused by different applications of the magnitude and timing's of muscle activities. It is shown experimentally in the reference ${ }^{6)}$ that the shapes of the characters are coded only in time, that is, it is the durations, not the magnitude, of muscle contraction that are coded, and that the magnitude of the force determines the general size of the characters written. Thus we assume that the control signal of the handwriting movements is specified by the square waveform having three magnitude levels as illustrated in Fig. 3, which we call control pulse. The

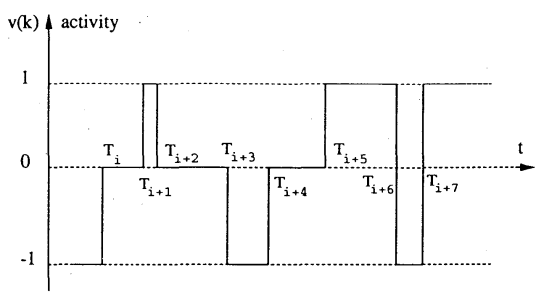

Fig. 3 Control pulse of handwriting movement
Fig. 2 Pulse controlled model of handwriting process

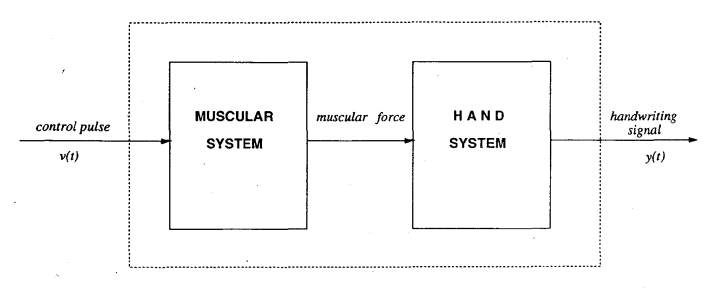

Fig. 2 Pulse controlled model of handwriting process 
levels 1 and -1 mean $\mathrm{ON}$ activities of the group of muscles to the positive and the negative directions, respectively, and 0 means $\mathrm{OFF}$ activity. $T_{i}$ 's are control-timing's at which the muscular activity changes from one level to another. It is the controltiming information, not the magnitude of the force used, that we do need to extract from the original handwritten characters.

The following notes must be added here as an important attribute for the present studies. The control-timing's defined above could be seen of quite abstract and somehow physiological fashion, that is, in the form of hypothetical neural motor commands that might be transmitted to the motor units of muscles to cause them to generate the prescribed muscular forces. By abstract we mean that the representations at the motor command level are very conceptual ones in the sense that there might exist no such signals in the actual human handwriting process. Since our goal of the present studies of the human handwriting process is not for obtaining precise physiological descriptions or guiding principles of the actual control mechanism of the process, but for extracting dynamic information sufficient enough for the on-line recognition of handwritten characters, the information extracted need not be so precise enough, but rather global in nature so as to be able to hold the least and sufficient features of the original handwritten characters and to make it easy for us to catch which characters are being written.

3. 3. 2 Expected roles of the control pulse

We think of the control pulses to be the representations at the motor command level shown in Fig. 1. They are expected to be more direct representations, namely, representations at the higher level of handwriting process than are the pencil-point movements, the importance of which was discussed in depth in the motor theory of speech perception by A.M. Liberman $^{2)}$.

It would be of help to give somewhat definitive discussions about how important roles the controltiming information play. For one of the typical examples, the discrimination between the cursive character る and み might not often be easy, because it is a matter of troubles not only to select features effective enough to discriminate between them, but to extract the features as well if we are to apply the traditional passive way of analysis. In other words, mapping each of the patterns onto the feature space of the limited dimension might result in big overlap. While, we hope that they are clearly separated each other in the feature space. This could be the case if the inverse transformation from the handwriting movements to the control-timing's has some nonlinear nature. The origin of the nonlinearity resides in how the control pulse is defined and in the algorithm applied to extract it. Note that our analysis method based on the MRACS does have this nonlinearity involved in its own framework, being discussed explicitly in the section 4.2. A closer examination of the control pulses concerning the above problem will be given along with the actual example in the section 5. 3 .

\section{4 Muscular System}

It can be supposed that the muscular force builds up and decays with certain transitional characteristics because of inter- or intra-muscular frictions and so forth. Thus, in order to shape the muscular force to have the characteristics mentioned above, it is necessary to introduce the muscular system $M$, a kind of filter, that receives the control pulse at the input and gives the muscular force at the output, see Fig. 2.

\section{5 Hand System ${ }^{7}$}

In order to have more generalized discussions on the dynamics of the handwriting movements, we consider a damped self-oscillation model schematized conceptually in Fig. 4, in which a mass point, the hand itself, is placed simply in the environment of viscosity $K_{d}$ through the spring of stiffness $N_{d}$ and makes forced self-oscillatory movements around a stable point when a pulsive force is applied. Note that the relevance of such a simplification of the model becomes possible from the assumption that the cursive handwriting has no feedback involved as mentioned in the beginning of this chapter. The motion of the mass point, when the muscle contracts by $\delta_{d}$, is expressed by the following 2nd order differential equation driven by the muscular force $F_{d}(t)$

$$
M \ddot{d}+2 K_{d} \dot{d}+N_{d} d=r \delta_{d}=F_{d}(t), \quad(d=x, y)
$$

where $M$ is the equivalent mass of the hand-pen combination.

By rewriting Eq. (1) in the form of difference equation, we have,

$$
d(k)=c_{1}^{d} d(k-1)+c_{2}^{d} d(k-2)+f^{d}(k),
$$

which is considered as the mathematical model of the hand system. Denoting the sampling interval by $h$, we have,

$$
\begin{aligned}
& c_{1}^{d}=\left(2-n_{d} h^{2}\right) /\left(1+k_{d} h\right), \\
& c_{2}^{d}=-\left(1-k_{d} h\right) /\left(1+k_{d} h\right), \\
& f^{d}(k)=F_{d}(k) h^{2} / M\left(1+k_{d} h\right), \\
& k_{d}=K_{d} / M, n_{d}=N_{d} / M .
\end{aligned}
$$

Eq. ( 2 ) states that the hand system can be expressed by the second-order auto-regressive (AR) model.

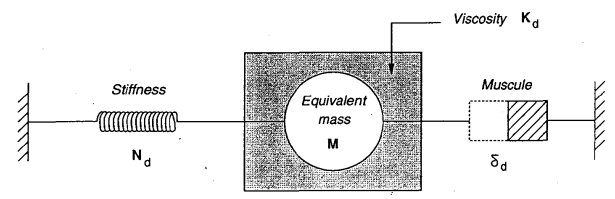

Fig. 4 Dynamic model of handwriting movement 


\section{6 Overall handwriting system}

The transfer function of the muscular system could be modeled by the moving average (MA) process, the all-zero model : $B\left(k, z^{-1}\right)$. This is because the effects of both the inter- and intra-muscular frictions are expected to result in certain energy absorption in some particular range of frequency components of the signal output from the muscular system. And, as mentioned in the previous section, the hand system is expressed by the AR model having the transfer function of the all-pole type: $1 / A\left(k, z^{-1}\right)$. These mean that the input-output relation of the overall handwriting system is given by,

$$
\begin{aligned}
& y(k+1)=\frac{B\left(k, z^{-1}\right)}{A\left(k, z^{-1}\right)} v(k)=H\left(k, z^{-1}\right) v(k), \\
& A\left(k, z^{-1}\right)=+a_{1}(k) z^{-1}+a_{2}(k) z^{-2} \\
&+\cdots+a_{m}(k) z^{-m} \\
& B\left(k, z^{-1}\right)= b_{0}(k)+b_{1}(k) z^{-1}+b_{2}(k) z^{-2} \\
&+\cdots+b_{n}(k) z^{-n}
\end{aligned}
$$

where $v(k)$ and $y(k+1)$ is the input control pulse and the output handwriting signal, respectively. $z^{-1}$ denotes the operator of a unit time delay. Eq. (7) states that the overall handwriting system can be expressed by the Auto-Regressive Moving Average (ARMA) model of the $(m, n)$ th order $(n<m)$. The ARMA-order is assumed actually to be $(2,1)$. Note that since both the stiffness and viscosity of the hand itself might be controlled through the spinal reflex ${ }^{5)}$, the parameters $a_{i}$ 's and $b_{i}$ s are assumed not stationary but slowly time-varying for the case of cursive handwriting.

\section{7 Validation of the modeling}

The basic idea of modeling human handwriting process described in this chapter was first presented by M. Yasuhara, one of the present authors, in $1983^{7}$. He proposed the 2nd order AR-model for the hand system $H$ based on the discussions given in the section 3.5 , and considered the problem of so-called blind deconvolution, that is, how to deconvolve the handwriting signal into two functions; the muscular force $m(t)$ and the impulse response $h(t)$ of the hand system, under the condition that the only data available is the convolved handwriting signal. The extensive comparative studies were also made to check its utilities between the muscular force function recovered and the electromyogram(EMG) signals obtained from the muscle Extensor Carpi Ulnaris for the horizontal wrist motion to the right and the muscle Abductor Pollicis Longus to the left ${ }^{1}$. He observed that the force functions recovered were in excellent agreement with the EMG signals, and further that the experiments gave stable and repeatable results for different texts and hands.

\footnotetext{
${ }^{1}$ These muscles play important roles in the respective principal component of the handwriting movements ${ }^{8)}$.
}

Although we need, of course, more thorough investigations on this problem, the above studies might be understood on the whole to give certain proof, though indirect and insufficient, to the statement that the proposed model is the realistic representation of the handwriting process.

\section{Extraction of Control Pulses}

It is the purpose of this chapter to show that it is possible to extract control pulses of handwriting movements based on MRACS theory and to describe how to apply the idea of the MRACS to our problem.

\subsection{The MRAC System}

Figure 5 shows the block diagram of Model Reference Adaptive Control System (MRACS) ${ }^{9)}$. The plant $H_{P}\left(k, z^{-1}\right)$, the target system to be controlled, is assumed to be a system having known system function with unknown time-varying parameters. The adjustable controller involves the same system function as that of the plant but the parameters are adjustable. The purpose of the MRACS is to adapt the coupled system of the controller and the plant, shown as the dashed box in the figure, to the reference model $H_{M}\left(k, z^{-1}\right)$ as well as to determine $u(k)$ in such a way that the output $y(k+1)$ from the plant follows $y_{M}(k+1)$ which is the output from the reference model.

\section{2 Stating the problem along with the MRACS}

Reference Model : To begin with, we suppose that the handwriting signals are composed of a sequence of segments having appropriate constant length. By the segment we mean a portion of the handwriting signal, during which the handwriting process is assumed stationary ${ }^{2}$, and consider the problem of segment-wise analysis of such signals as could be modeled to be the output $y_{M}(k+1)$ from the following

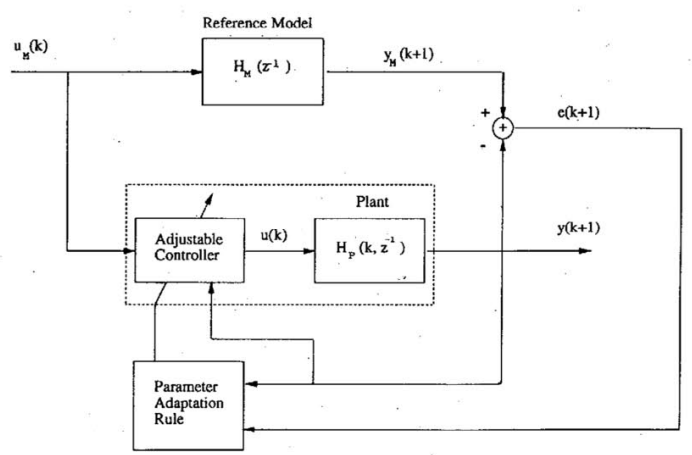

Fig. 5 Model reference adaptive control system

\footnotetext{
${ }^{2}$ The handwriting process is not generally considered stationary, but varies slowly as far as the cursive handwriting process is concerned because it has no rapid feedback process involved.
} 
pole-zero system, the reference model, driven by the hypothesized input $u_{M}(k)$ as shown in Fig. 6,

$$
\begin{aligned}
y_{M}(k+1)= & H_{M}\left(z^{-1}\right) u_{M}(k)=\frac{B_{M}\left(z^{-1}\right)}{A_{M}\left(z^{-1}\right)} u_{M}(k), \\
A_{M}\left(z^{-1}\right)= & +a_{M 1} z^{-1}+a_{M 2} z^{-2} \\
& +\cdots+a_{M_{m}} z^{-m} \\
B_{M}\left(z^{-1}\right)= & b_{M 0}+b_{M 1} z^{-1}+b_{M 2} z^{-2} \\
& +\cdots+b_{M n} z^{-n}
\end{aligned}
$$

The characteristic equation of $A_{M}\left(z^{-1}\right)$ must have all its roots within a unit circle on the $z$-plane with its center at the origin. The reference model is assumed to be known and fixed during the same segment. Note that $y_{M}(k+1)$ is actually the handwriting signal itself to be analyzed.

Plant: As shown in Fig. 7, the handwriting signal synthesized is understood to be the output from the plant driven by $u(k)$ at the input. Further, we think that the plant is divided into two subsystems; a threshold element $T$ and a synthetic model $H_{s}\left(z^{-1}\right)$ of the handwriting, given as,

$$
T(x)=\left\{\begin{aligned}
1, & \text { for } x \geq \theta \\
0, & \text { for }|x|<\theta \\
-1, & \text { for } x \leq-\theta,
\end{aligned}\right.
$$

where $\theta$ is the predetermined threshold value, and

$$
\begin{aligned}
& H_{S}\left(z^{-1}\right)=\frac{B_{S}\left(z^{-1}\right)}{A_{s}\left(z^{-1}\right)}, \\
& A_{s}\left(z^{-1}\right)=1+a_{s 1} z^{-1}+a_{s 2} z^{-2}+\cdots+a_{s m} z^{-m}, \\
& B_{S}\left(z^{-1}\right)=b_{S 0}+b_{s 1} z^{-1}+b_{s 2} z^{-2}+\cdots+b_{s n} z^{-n} .
\end{aligned}
$$

The output of $T$ corresponds to the control pulse $v(k)$ which is the target to be extracted in the problem. Our final goal is not to estimate $u(k)$ but to extract $v(k)$. It follows, therefore, that it is necessary to set $H_{S}\left(z^{-1}\right)$ equal to $H_{M}\left(z^{-1}\right)$, that is, $a_{s i}=a_{M i}(i=1,2, \cdots$, $m)$ and $b_{s j}=b_{M j}(j=0,1, \cdots, n)$. Thus we have $H_{P}=$ $H_{T} H_{M}\left(z^{-1}\right)$, where $H_{T}$ is the system function of the threshold element $T . H_{P}$ must be nonlinear since $H_{T}$ is nonlinear. The problem here is that the MRACS theory cannot be applied because of the nonlinearity of $H_{P}$. We are to overcome this problem by introducing the assumption that $H_{P}$ is of the $(m, n)$-order ARMA model having unknown time-varying parameters as follows,

$$
\begin{aligned}
& H_{P}\left(k, z^{-1}\right)=\frac{B_{P}\left(k, z^{-1}\right)}{A_{P}\left(k, z^{-1}\right)}, \\
& A_{P}\left(k, z^{-1}\right)=1+a_{P 1}(k) z^{-1}+a_{P 2}(k) z^{-2}+\cdots \\
& \stackrel{\mathfrak{u}_{M}(\mathbf{k})}{\longrightarrow} \frac{\mathrm{B}_{\mathrm{M}}\left(\mathbf{z}^{-1}\right)}{\mathbf{A}_{M}\left(\mathbf{z}^{-1}\right)} \stackrel{\mathrm{y}_{M}(\mathbf{k}+1)}{\longrightarrow}
\end{aligned}
$$

Fig. 6 Supposed model of the signal process

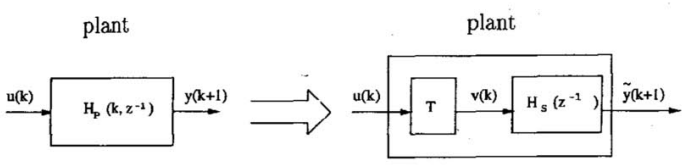

Fig. 7 Structure of the Plant

$$
\begin{aligned}
& +a_{P m}(k) z^{-m}, \\
B_{P}\left(k, z^{-1}\right)= & b_{P 0}(k)+b_{P 1}(k) z^{-1}+b_{P 2}(k) z^{-2}+\cdots \\
& +b_{P n}(k) z^{-n} .
\end{aligned}
$$

It follows, therefore, that we have replaced the nonlinear system by the time-varying linear one.

As the result, although both $H_{T}$ and $H_{S}\left(z^{-1}\right)$ are known and fixed together within the segment, the system function of the coupled system, the plant, must be assumed unknown time-varying within that segment. This is the reason why adaptive control system must be applied to our problem. The problem is to determine $u(k)$ such that the output $y(k+1)$ of the plant follows the given signal $y_{M}(k+1)$ as closely as possible under the condition that the plant parameters are unknown time-varying. The MRACS theory makes it possible for us to solve this problem.

\section{3 System Theoretic Representation of the Plant}

At first, we define $(n+m)$ dimensional plant state vector as,

$$
Y_{0}^{t}(k)=[u(k-1) \cdots u(k-n) \mid y(k) \cdots y(k-m+1)] .
$$

$t$ indicates to transpose. Based on this state vector, we consider the input-output relation of the plant to be updated according to the following system,

$$
\begin{aligned}
& Y_{0}(k+2)=G Y_{0}(k+1)+I I u(k+1), \\
& \tilde{y}(k+1)=C Y_{0}(k+1)+b_{s 0} q(k),
\end{aligned}
$$

where $G$ is a $(n+m) \times(n+m)$ matrix defined as,

$$
\begin{aligned}
& G=
\end{aligned}
$$

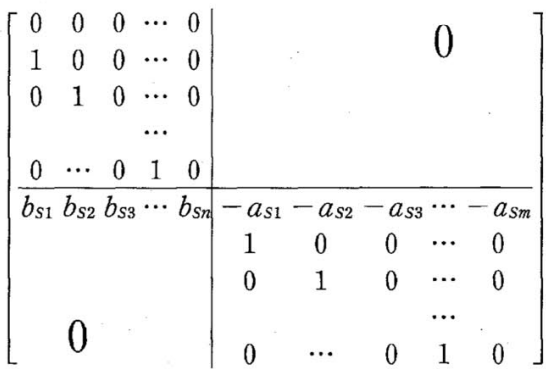

and,

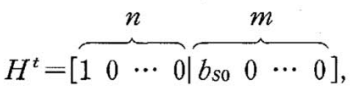

$$
\begin{aligned}
& C=\left[\begin{array}{llllllll}
0 & 0 & \cdots & 0 & 0 & \cdots & \cdots
\end{array}\right],
\end{aligned}
$$

$$
q(k)=v(k)-u(k), E[q(k) q(l)]=\delta_{k l} \sigma_{q}^{2},
$$

where $E[\cdot]$ means to take expectation. It follows that $Y_{0}(k+1)$ is observable only through Eq. (22). $q(k)$ corresponds to the estimation error of $v(k)$, incurred by the threshold element, which is assumed white and independent to $u(k)$. Eq. (21) and Eq. (22) constitute together the system-theoretic representation of the plant, the block diagram of which is shown in Fig. 8. 


\section{4 Construction of the System Based on MRACS}

State Observer : In order to formulate our problem along with the MRACS algorithm presented in the appendix $\mathrm{B}$, the plant state vector $Y_{0}(k+1)$ must be assumed to be known. However, since the plant outpui $\widetilde{y}(k+1)$ given by Eq. (22) is the only element that is observable, our next problem is to make an optimum estimate of the whole elements of the plant state vector under the condition that a noisy one of its elements is observable. Thus a state observer is constructed to have the optimum estimate of $Y_{0}(k$ $+1)$, the algorithm of which is well-known and derived easily from that of the Kalman filter by taking Eq. (21) and Eq. (22) for the signal and the observation processes, respectively.

System Construction: Based on this optimum state vector estimated, $u(k)$ is calculated and the controller parameters are updated according to the MRACS algorithm so that the controller-plant couple may follow the reference model. Figure 9 shows the whole system block diagram of the active analysis in the final form. The system constitutes totally an adaptive control process that calculates the optimum input $u(k)$ to the plant, the dashed box, such that its output $\widehat{y}(k+1)$ follows the given signal $y_{M}(k+1)$. Note that the state observer is embedded in the final form of the plant. The whole body of the plant including the state observer appears eventually to be unknown time-varying system when viewed from the outside.

\section{Experimental Studies}

Although the ultimate goal of our investigations is

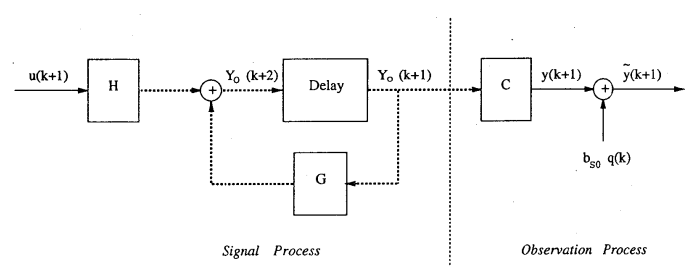

Fig. 8 System theoretic representation of the Plant

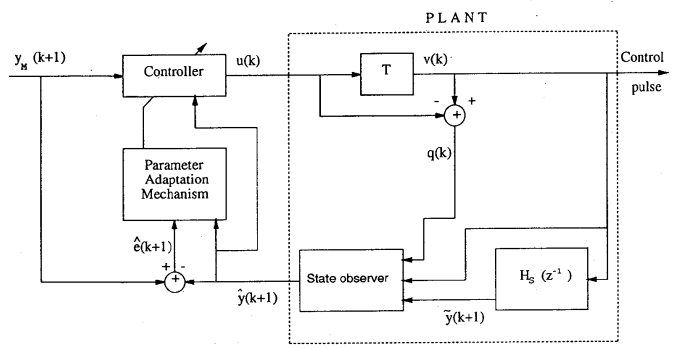

Fig. 9 Block diagram of the active analysis based on MRACS in the final form to propose an active recognition scheme to the on-line recognition of cursively handwritten characters, the present paper is not concerned directly with this end, but with having theoretical considerations about the problem in depth and giving the mathematical basis to realize the idea proposed. Thus, the experimental data reported in this chapter are not tested under the actual condition based on the final recognition experiments. The purpose of this chapter is to check experimentally the feasibilities of the method proposed to extract the control pulse of human handwriting movements. Several computer simulation runs have been conducted to give some ideas of the performances.

\section{1 Experimental Conditions Assumed}

Several important experimental conditions assumed are summerized in the following.

Data Base: The original pen-point movement signals $x_{M}(k)$ and $y_{M}(k)$ from the writing tablet are sampled at $32 \mathrm{~Hz}$ into 12-bit digital samples (30 dots $/ \mathrm{mm}$ ) and applied for the analysis.

Initial Values: The initial value of the parameter vector $P(k)$ (see appendix B) of the controller, and the initial value of the plant state vector must be given before the program can run the extraction process of the control pulse. The controller parameter vector $P(k)$ is required to be initialized on segment-by-segment basis to the new value estimated from the batch data of the signal segment by use of the blind deconvolution technique developed by $\mathrm{M}$. Yasuhara $^{7)}$. We assume the initial values of the controller parameter vector and the plant state vector as,

$$
P^{t}(m)=\left[b_{0}(m) P_{0}^{t}(m)\right]=\left[b_{M 0} \cdots b_{M n} \mid-a_{M 1} \cdots-a_{M m}\right],
$$

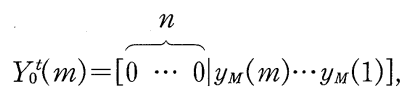

where $a_{M i}(i=1,2, \cdots, m)$ and $b_{M j}(j=0,1, \cdots, n)$ are the estimated values mentioned above.

Threshold Element ${ }^{3}$ : If the distribution density of $u(k)$ could be assumed Gaussian, The threshold value of $\theta=0.6120$ might be applied based on 3-level Gaussian optimum quantizer ${ }^{10)}$. However there is no positive reason for the Gaussian assumption of $u(k)$, our strategy taken for this feasibility study is to search the best value around 0.6120 .

Performance Criterion: Of course, the performance must be evaluated based on the final recognition rate attained. But, instead, we use here the measure of signal-to-distorsion ratio (SDR) to judge the feasibility of the proposed scheme. The SDR is defined in $\mathrm{dB}$ on stroke-by-stroke basis by

$$
S D R=10 \log
$$

${ }^{3}$ The threshold value $\theta$ must be chosen properly based on the actual recognition experiments. 


$$
\times \frac{\left\langle x_{M}^{2}(k)+y_{M}^{2}(k)\right\rangle}{\left\langle\left(x_{M}(k)-\widehat{x}(k)\right)^{2}+\left(y_{M}(k)-\widehat{y}(k)\right)^{2}\right\rangle},
$$

where $\hat{x}(k)$ and $\hat{y}(k)$ denote the synthesized signals and $\langle\cdot\rangle$ means averaging over the stroke in question. By the stroke we mean a segment of handwriting signal during which the pen-point touches on the writing surface. All the signals are assumed to have zero mean.

\section{2 Some Experimental Results}

Figure 10 shows one of the typical examples of executing the control pulse extraction for the fast cursive handwriting sample "yes" (written in about 1 sec.). Note, for this particular example, that the impulse response $m(t)$ of the muscular system $M$ for the $y$-signal is of the damped oscillator with the resonance frequency of $2.43 \mathrm{~Hz}$, which was calculated by using the blind deconvolution technique. This means that the output $y$-signal has the damped selfoscillatory component of $2.43 \mathrm{~Hz}$ when excited by a single impulse at the input.

Because of the assumption of such a self-oscillatory model, it follows that no control pulses must be extracted before the oscillatory component of the output signal cannot follow the given $y$-signal, in other words, a control pulse occurs only at the time when the synthesized trace of the pencil-point movement comes up with following incorrectly the trace of the actual handwriting movement given. These are originated from the characteristic properties of the model applied here, the ARMA model.

Keep the comments above in mind, and careful inspections of Fig. 10 will reveal that fairly reasonable control pulses have been extracted. The extensive experimental studies have also shown stable and repeatable results of extracting the control pulse sequences for different texts and hands.

Next, an example is shown in Fig. 11 for the sample written very slowly in about 3 second. Note that extra small pulses are extracted in this case. These situations were found quite common throughout the

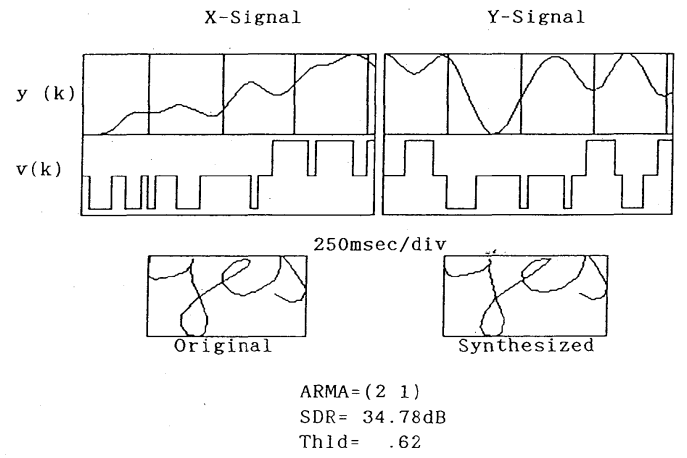

Fig. 10 Experiment for fast handwriting extensive comparative studies between the fast cursively and the slowly handwritten samples. The control pulses extracted for the slow handwriting can not be made to have similar properties as those for the fast handwriting without applying higher threshold value of $\theta$. Applying the higher threshold value $\theta$ decreases the number of small extra pulses, but reduces the system performances judged by SDR. Figure 12 shows that the best SDR is attained at $\theta=$ 0.49 in the case of Fig. 10. Note of course that the proper value of $\theta$ must be selected based on the actual recognition experiments. One of the possible accounts of the extra small pulses might be given as being caused by the positional feedback involved because the sample was written so slowly that the spinal reflex or the feedback through the eye had enough time to control the movements precisely.

\section{3 Utility of the active recognition - an exam- ple}

As mentioned in the section 3.3.2, we have many set of such characters that the distinction among them might often be difficult. As one of the typical examples, Fig. 13 shows the comparison of two experiments extracting the control pulses for each of the handwritten characters る and み, in which $\theta=1.0$ was chosen. In the case of (a), there is a general stop of the pencil-point movement around $700 \mathrm{msec}$.

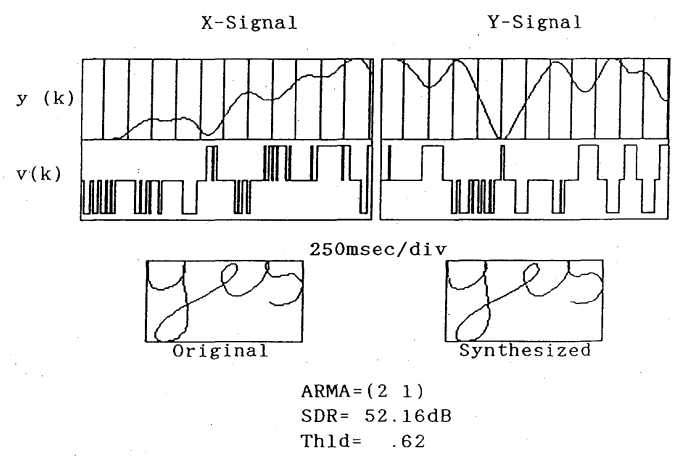

Fig. 11 Experiment for slow handwriting

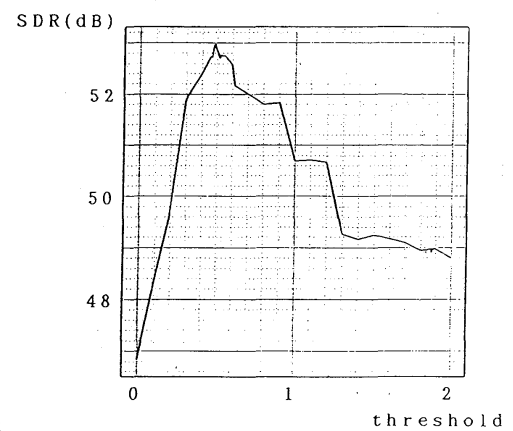

Fig. 12 Effect on the SDR of the threshold $\theta$ for the sample of the Fig. 10 


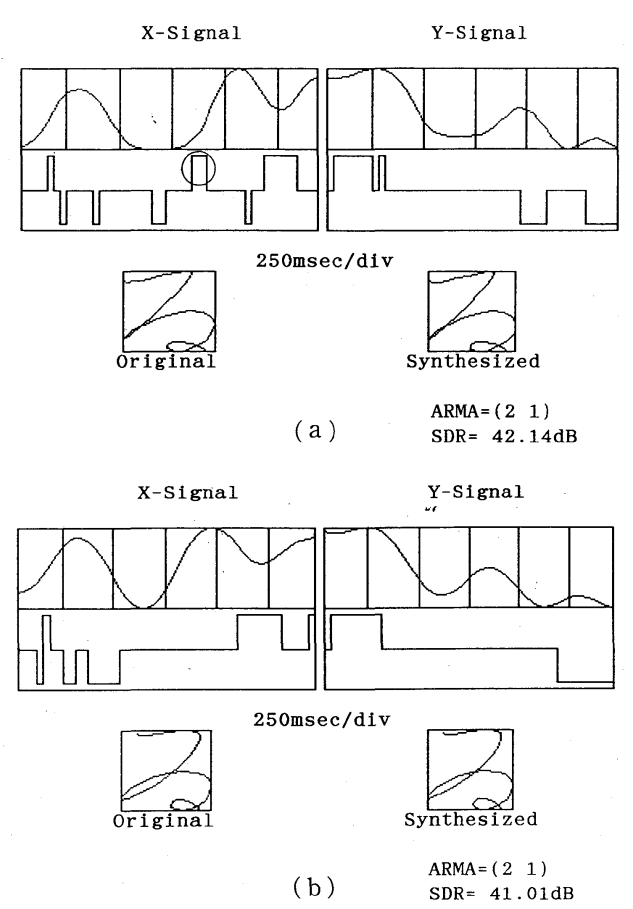

Fig. 13 Comparison of the extracted control pulse for the two cases: ( a ) る and ( b ) み

from the start of writing, but no one in the case of (b). By the general stop we mean the time period during which the pencil-point does not move at all the stopping period might be short or long depending on the cases.

On the first hand, it must be impossible to identify the existence of such general stop of the handwriting movement as far as only the static character images are concerned. On the other hand, even if the time waveforms of the handwriting movements are available, such situations must be noted also where it is difficult to catch whether or not there exists any general stop because of the presence of various kinds of noises.

Careful inspections of the extracted control pulse of the case (a) reveal that there we have a positive pulse - marked by the circle in the figure - in the Xdirection just after the general stop of the movement. While, we cannot find any one around there in the case (b). Referring to the comments given in the section 5.2 , this could be understood such that the pulse might be a motor command transmitted to the motor units of muscles to cause them to generate the muscular force to make the hand-pen couple move to the positive $\mathrm{X}$-direction just after the general stop. Thus the existence of such pulse can tell clearly that the character belongs to $る$. Note that there exists no such pulse for the Y-direction, because the pencil- point moves neither so slowly nor quickly to the Ydirection, in other words, the speed of the movement may be quite near to that of the self-oscillatory movement being output from the model.

The inherent nonlinear property of the proposed active analysis based on the MRACS could extract such pulses with good stability. More specifically, the threshold element involved in the system could be expected to make it possible to detect the pulses even in the presence of noise when the threshold value $\theta$ is chosen properly.

\section{Conclusions and Some Remarks}

Aiming at the on-line recognition of cursive handwritten characters, we have introduced the concept of active recognition, and presented the actual technique to realize it. To begin with, the pulse-controlled model of handwriting movement was proposed based on the ARMA process. Then the method to extract the control pulses from the handwriting signal as well as its mathematical basis have been presented based on the model reference adaptive control system (MRACS). Finally it has been demonstrated experimentally that our method is able to be used successfully.

Although our method presented here is not tested under the final recognition experiments, the ARMA process applied to modeling the human handwriting had been validated in the previous paper by one of the authors as discussed in the section 3.7, and the possibility has been demonstrated that the method can tell clearly the delicate differences between characters of difficult distinction. And further, we should point out herein the important experimental fact that quite the similar and stable patterns of control pulse sequences are extracted for the movements intending to write the characters of the same category.

Further studies are concerned with the execution of the active recognition of the cursive handwritten characters by use of the method presented here. Investigations are underway in this direction and will be reported by the separate paper in due time.

It must be kept in mind again that it is not necessary for the recognition of handprinted characters to apply such a complicated active method and to extract signals at the higher level of handwriting process, that is, a simpler and straightforward method based on the passive analysis can be used successfully which converts the handprinted characters directly to the corresponding symbols.

Finally, it may be worthwhile to add that the presented method to extract the control pulse can also be applicable, for example, to estimating impulses of vocal tract excitation in speech generation process. 


\section{References}

1) K. N. Stevens: Toward a Model for Speech Recognition, Jour. Accoust. Soc. Amer., 32, 47/51 (1960)

2) A. M. Liberman and I. G. Mattingly: The Motor Theory of Speech Perception, Cognition, 21, 1/36 (1980)

3) T. Huang and M. Yasuhara: Recovery of Drawing Order Information of Cursive Scripts of Single Stroke from their $2 \mathrm{D}$ Images, tobe publised on Trans. of Information Processing Society of Japan. (1995)

4) K. S. Lashley: The Accuracy of Movements in the Absence of Excitation from the Moving Organ, Amer. Jour. Physiol., 43, 169/194 (1917)

5) G.D. Dawson: Cerebral response to nerve stimulation in Man, Brit. Med. Bull., 6, 326/329 (1950)

6) M. Yasuhara: Experimental Studies of Handwriting Process, Rep. Univ. Electro-Comm., 25-2, 233/254 (1975)

7) M. Yasuhara: Identification and Decomposition of Fast Handwriting System, IEEE Trans., CAS-30-11, 828/ 832 (1983)

8) T. von Lanz and W. Wachsmuth: Praktische Anatomie Arm, Springer 1959, Berlin Germany.

9) I. D. Landau and M. Tomizuka: Theory and Practice of Adaptive Control System, (in Japanese), Ohm-Sha, (1981)

10) J. Max: Quantizing for Minimum Distortion, IRE Trans. IT-6-3, 7/12 (1960)

11) M. Eden and M. Halle: The Characterization of Cursive Writing, Proc. 4th London Sym. on Information Theory, 287/299 (1961)

12) D. Van der Gon and J. Thuring: A Handwriting Simulator, Physiol. Med. Biol., 6, 407/414 (1962)

13) J. MacDonald : Experimental Studies of Handwriting signals, Tech. Rep. 443 MIT (1966)

14) J. M. Hollerbach: An Oscillation Theory of Handwriting, Biol. Cybern., 39, 139/156 (1981)

15) M. Eden and P. Mermelstein: Mathematical Models for the Dynamics of Handwriting Generation, Proc. 16th Annual Conf. on Engineering in Medicine and Biology, Baltimore, Maryland, 12/13 (1983)

16) S. Edelman and T. Flash: A Model of Handwriting, Biol. Cybern., 57, 25/36 (1987)

17) R. Plamondon: A Theory of Rapid movements, In G. E. Stelmach, J. Requin (eds) Tutorials in Motor Behavior II, Elsevier, Amsterdam, 55/69 (1992)

18) B. Simard, B. Prasada and R. M. K. Sinha : On-line Character Recognition Using Handwriting Modeling, Pattern Recognition, Pergamon Press, 26-7, 993/1007 (1993)

19) Y. Wada, Y. Koike and M. Kawato: A Computational Model of Cursive Handwriting, Trans. IEICE, J 76-DII11, 2400/2410 (1993)

\section{Appendix}

\section{A. Comments on the Studies Concerning Gener- ation of Human Handwriting}

The references cited in (6) and in (11)-(19) are the papers dedicated to the studies of human handwriting generation. They are contributory to our concept of an active recognition of cursive characters. Though this kind of research works has been relatively rare to be found, among them, the recent works (14) - (19) are very interesting from the stand- ing point of the current study.

B. MRAC algorithm for the $(m, n)$-order ARMA model

(1) Reference model:

$A_{M}\left(z^{-1}\right) y_{M}(k+1)=B_{M}\left(z^{-1}\right) u_{M}(k)$.

(2) Controller parameter to be estimated:

$$
\begin{aligned}
& P^{t}(k)=\left[b_{0}(k) P_{0}^{t}(k)\right], \\
& \left.P_{0}^{t}(k)=\left[b_{1}(k) \cdots b_{n} k\right) \mid-a_{1}(k) \cdots-a_{m}(k)\right] .
\end{aligned}
$$

(3) Control input:

$$
u(k)=\left\{y_{M}(k+1)-Y_{0}^{t}(k) P_{0}(k)\right\} / b_{0}(k) \text {. }
$$

(4) Plant system :

$$
A_{P}\left(k, z^{-1}\right) y(k+1)=B_{P}\left(k, z^{-1}\right) u(k) \text {. }
$$

(5) Plant state vector:

$$
\begin{aligned}
& Y^{t}(k)=\left[u(k) Y_{0}^{t}(k)\right], \\
& Y_{0}^{t}(k)=[u(k-1) \cdots u(k-n) \mid y(k) \cdots y(k-m+1)] .
\end{aligned}
$$

(6) Adaptive error signal :

$$
\begin{aligned}
& e^{*}(k+1)=\frac{y(k+1)-Y^{t}(k) P(k)}{1+Y^{t}(k) F(k) Y(k)} . \\
& (7) \quad \text { Adaptation rule: } \\
& P(k+1)=P(k)+F(k) Y(k) e^{*}(k+1) .
\end{aligned}
$$

(8) Adaptation gain:

$$
\begin{aligned}
F(k+1)= & \frac{1}{\lambda_{1}(k)} \\
& \times\left\{F(k)-\frac{\lambda_{2}(k) F(k) Y(k) Y^{t}(k) F(k)}{\lambda_{1}(k)-\lambda_{2}(k) Y^{t}(k) F(k) Y(k)}\right\} \\
& 0<\lambda_{1}(k) \leq 1,0 \leq \lambda_{2}(k)<2, F(m)>0 .
\end{aligned}
$$

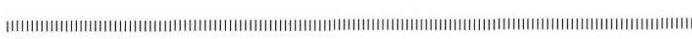
Youssef IGuider

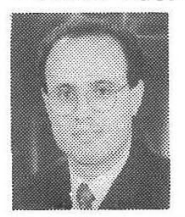

Was born in Morocco in 1961. He received the M. Sc. in Electrical Engineering from the Odessa Polytechnic Institute in USSR in 1987. He is involved in the Doctoral program with the Department of Computer Science and Information Mathematics at the University of ElectroCommuniccations in Tokyo since April 1990. Currenty he is on leave to work with the R\&D Center at Kyushu Matsushita Electric co. His research interests include handwriting recognition and its applications.

Makoto Yasuhara（Member）

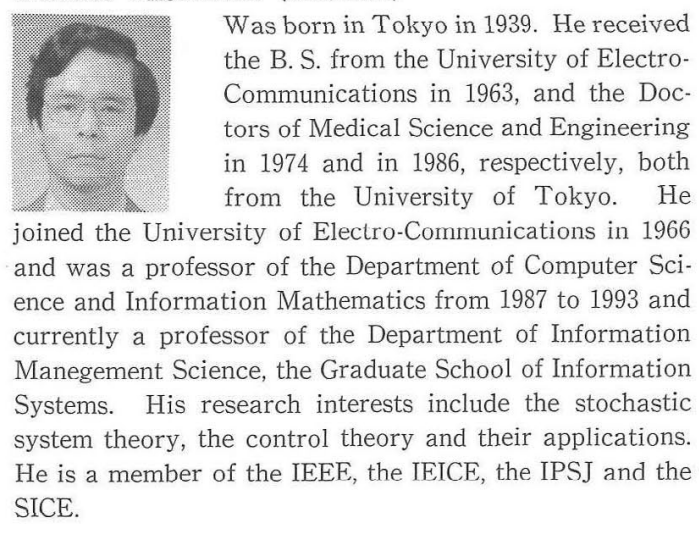

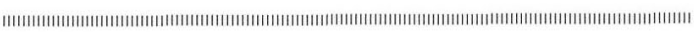

